\title{
Estudo do Processo TIG Hot-Wire com Material de Adição AISI-316L Analisando o Efeito do Sopro Magnético do Arco sobre a Diluição do Cordão de Solda
}

\author{
Erick Alejandro González Olivares ${ }^{1}$, Victor Manuel Vergara Díaz² \\ 1 Welding and Joining Institute, RWTH Aachen University, Aachen, Germany. \\ 2 Universidad de Antofagasta, Departamento de Ingeniería Mecánica, Antofagasta, Chile.
}

Recebido: 02 Mar., 2016

Aceito: 10 Ago., 2016

E-mails: erick.gonzalez.olivares@rwth-aachen.de (EAGO), victor.vergara@ uantof.cl (VMVD)
Este é um artigo publicado em acesso aberto (Open Access) sob a licença Creative Commons Attribution Non-Commercial, que permite uso, distribuição e reprodução em qualquer meio, sem restriçōes desde que sem fins comerciais e que 0 trabalho original seja corretamente citado.
Resumo: O processo TIG com adição de arame quente (HotWire) é considerado um processo altamente produtivo em comparação ao processo TIG convencional com arame frio (ColdWire), sendo possível alcançar grandes taxas de deposição e baixas porcentagens de diluição, características essas que permitem ao processo ser uma ótima alternativa para aplicações de revestimentos. Existem variadas opções em configurações de circuitos elétricos para aquecer o arame de adição no processo TIG Hot-Wire, entre elas podemos destacar a utilização da corrente contínua pulsada e a corrente alternada. No presente trabalho foi utilizada a corrente contínua constante e foram estudadas duas configurações do circuito elétrico para aquecer o material de adição, as quais em teoria provocam diferentes comportamentos no arco voltaico e, portanto, nas propriedades geométricas do cordão de solda. Uma primeira análise realizada sobre o arco voltaico demonstra que se forma um sopro mágnetico constante ao se aquecer o arame com corrente contínua constante. Segundo os ensaios realizados, a direção do sopro magnético depende da polaridade da corrente para aquecer o arame. Uma análise macrográfica dos cordões de solda obtidos demonstraram que os melhores resultados foram para os ensaios feitos com Hot-Wire, conseguindo diluições de até um $2 \%$ aproximadamente para uma velocidade de arame de 7,5 m/min, $1 \mathrm{~kW}$ de potência de aquecimento do arame e usando uma velocidade de soldagem de $30 \mathrm{~cm} / \mathrm{min}$.

Palavras-chave: TIG; Hot-Wire; Sopro magnético; Diluição.

\section{Study of the TIG Hot-Wire Process Using AISI-316L like Addition Material Analyzing the Effect of the Magnetic Blow Arc over Weld Bead Dilution}

\begin{abstract}
The TIG process with Hot-Wire addition is described as a process highly productive, in comparison with conventional TIG process with Cold-Wire, achieved large deposition rates and low dilution percentages, characteristics that permit to the process to be an optimum alternative for cladding applications. There are many options of electrical circuits configurations for to heat the addition wire in the TIG Hot-Wire process, highlighting the utilization of the continuous pulsed current and the alternating current. In the present paper were studied two configurations of the electrical circuit for to heat the addition materials, whom in theory provoke different behaviors in the welding arc, and therefore, in the geometrical properties of the welding bead too. A first analysis done on the welding arc, demonstrate that to heat the wire with continuous constant current is generated a permanent magnetic blow over the arc. According to trials made, the direction and intensity of the magnetic blow will depend of the polarity and of the current value for to heat the wire. A macrographic analysis of the weld beads demonstrated that the best results were for the tests made with Hot-Wire, achieving dilutions until a $2 \%$ for a wire velocity of $7,5 \mathrm{~m} / \mathrm{min}, 1 \mathrm{~kW}$ of power for to heat the wire and it using a welding velocity of $30 \mathrm{~cm} / \mathrm{min}$.
\end{abstract}

Key-words: TIG; Hot-Wire; Magnetic blow; Dilution.

\section{Introdução}

Atualmente no mercado existem vários processos para se realizar um revestimento metálico. Dentro dos processos de soldagem que se destacam para realizar esta tarefa encontram-se o MIG/MAG, TIG e o PTA. O processo MIG/MAG oferece uma grande produtividade, mas o aparecimento de respingos sobre o metal de base e os consumíveis da tocha faz com que a qualidade superficial do revestimento seja afetada, além disso, o processo MIG/MAG não se sobressai por possuir um baixo nível de diluição. No caso do processo PTA, ele possui como principal aplicação a realização de revestimentos, devido aos seus baixos níveis de diluição (menores a um 5\%) [1], ele acaba por possuir um excelente 
Estudo do Processo TIG Hot-Wire com Material de Adição AISI-316L Analisando o Efeito do Sopro Magnético do Arco sobre a Diluição do Cordão de Solda

acabamento superficial e geralmente não precisa um processo mecanizado de pós-soldagem. No processo PTA, é possível trabalhar tanto com material de aporte em formato de pó ou arame, conseguindo os melhores resultados com pó. Porém, o processo PTA não é capaz de gerar uma grande produtividade, por possuir muitos parâmetros a ser controlados faz com que pequenas variações acabem por afetar imediatamente a qualidade do revestimento. Por outro lado, o processo de soldagem TIG convencional não é considerado produtivo devido à sua baixa taxa de deposição, em comparação ao processo MIG/MAG, porém, ele tem um excelente acabamento superficial, livre de respingos. Nos trabalhos feitos por Silva et al. [2] e Miranda [3] usando o processo TIG com adição de arame frio (Cold-Wire) para fazer revestimentos com Inconel 625, foi possível conseguir porcentagens de diluição ao redor de $16 \%$, com uma excelente qualidade superficial para uma velocidade de arame de $6 \mathrm{~m} / \mathrm{min}$ e uma velocidade de soldagem de $25 \mathrm{~cm} / \mathrm{min}$.

Na década dos anos 60 foi desenvolvida a técnica chamada "Hot-Wire" [4], com o objetivo de aumentar a taxa de deposição do processo TIG [5]. Em uma aplicação normal do processo de soldagem TIG Cold-Wire, o material de adição frio é fornecido na borda da poça de fusão, fazendo com que o arco voltaico perca eficiência devido à sua entrega de energia para poder fundir o material de aporte, além de fornecer energia para manter a poça de fusão no material de base. No caso da soldagem TIG com a técnica "Hot-Wire", o arame é considerado uma resistência para o fluxo da corrente elétrica provocando assim um aquecimento do arame, facilitando dessa forma a fusão dele no momento de ser depositado na poça de fusão, geralmente é introduzido por de trás do eletrodo de tungstênio [4]. Existem inúmeros métodos pelos quais pode ser aquecido o arame, o mais simples deles é circular uma corrente constante pelo material de adição, que será aquecido ao fechar o circuito elétrico em contato com a poça fundida (Efeito Joule). A desvantagem dessa forma de aquecimento, segundo a literatura consultada [4-6], é o forte sopro magnético que afeta ao arco voltaico, devido ao campo magnético produzido pela corrente que circula no arame de adição. Como solução a esse problema, fontes de corrente alternada são usadas, dessa forma a alternância entre a polaridade negativa e positiva a uma determinada frequência, diminuem consideravelmente o sopro magnético [6]. No trabalho de Shinozaki et al. [7] destaca-se outro sistema de aquecimento do arame, que também trabalha com o princípio de efeito Joule, mas nesse caso utilizaram corrente pulsada em uma determinada frequência e polaridade, com o objetivo de eliminar alguns problemas, principalmente o sopro magnético do arco. Segundo a informação disponibilizada pelo CCWJ [8], eles também utilizam uma corrente pulsada para aquecer o arame, porém, é possível notar que a corrente usada durante o período de base é bastante baixa, devido ao menor sopro magnético sobre o arco voltaico. Dessa forma, eles obtêm um grande controle sobre o material depositado, trabalhando com uma velocidade de arame de $6 \mathrm{~m} / \mathrm{min}$ e uma velocidade de soldagem de $50 \mathrm{~cm} / \mathrm{min}$.

Se faz necessário destacar que os métodos de aquecimento de arame nomeados só podem ser aplicados a materiais que possuam uma alta resistividade elétrica, restringindo seu uso a materiais como o cobre ou alumínio. Porém, nos trabalhos desenvolvidos por Lv et al. $[9,10]$ descreve-se a aplicação de um arco voltaico extra, dedicado unicamente ao aquecimento do arame, alcançando mediante esse método temperaturas de até um $60 \%$ do ponto de fusão.

O sopro magnético é considerado um defeito sobre o arco voltaico, e por tanto, deve ser evitado a todo momento. Este defeito é formado por distúrbios magnéticos (campos) perto do arco voltaico, que provocam o desvio e diminuição no controle do jato de plasma [11-13]. Como foi mencionado anteriormente, o processo TIG Hot-Wire na sua forma mais simples, com corrente contínua constante, produz um sopro magnético sobre o arco voltaico [5-7,9]. Porém, no trabalho realizado por Burt [14] foi utilizado um sistema de aquecimento do arame com corrente alternada de baixa frequência com o propósito de provocar uma pequena oscilação no arco voltaico mediante o sopro magnético, conseguindo diluições inferiores ao $8 \%$ com uma taxa de deposição de $6,43 \mathrm{~kg} / \mathrm{h}$, demonstrando assim que o sopro magnético pode ser benéfico, em certas condições. Por outro lado, Reis [15] estudou o comportamento do processo MIG/MAG Tandem, e especificamente a sua interação entre os campos magnéticos produzidos por dois arcos voltaicos, explicando desta forma a direção que toma o arco voltaico ante a presença de um campo magnético externo. Na Figura 1 encontra-se um esquema adaptado do trabalho de Reis [15], onde é possível observar como um arco voltaico sofre uma mudança no comportamento devido à exposição de um campo magnético externo. Através do exposto por Reis [15], Schwedersky [16] conseguiu explicar as instabilidades para certas condições de trabalho no estudo desenvolvido com o processo TIG duplo eletrodo (Figura 2). 

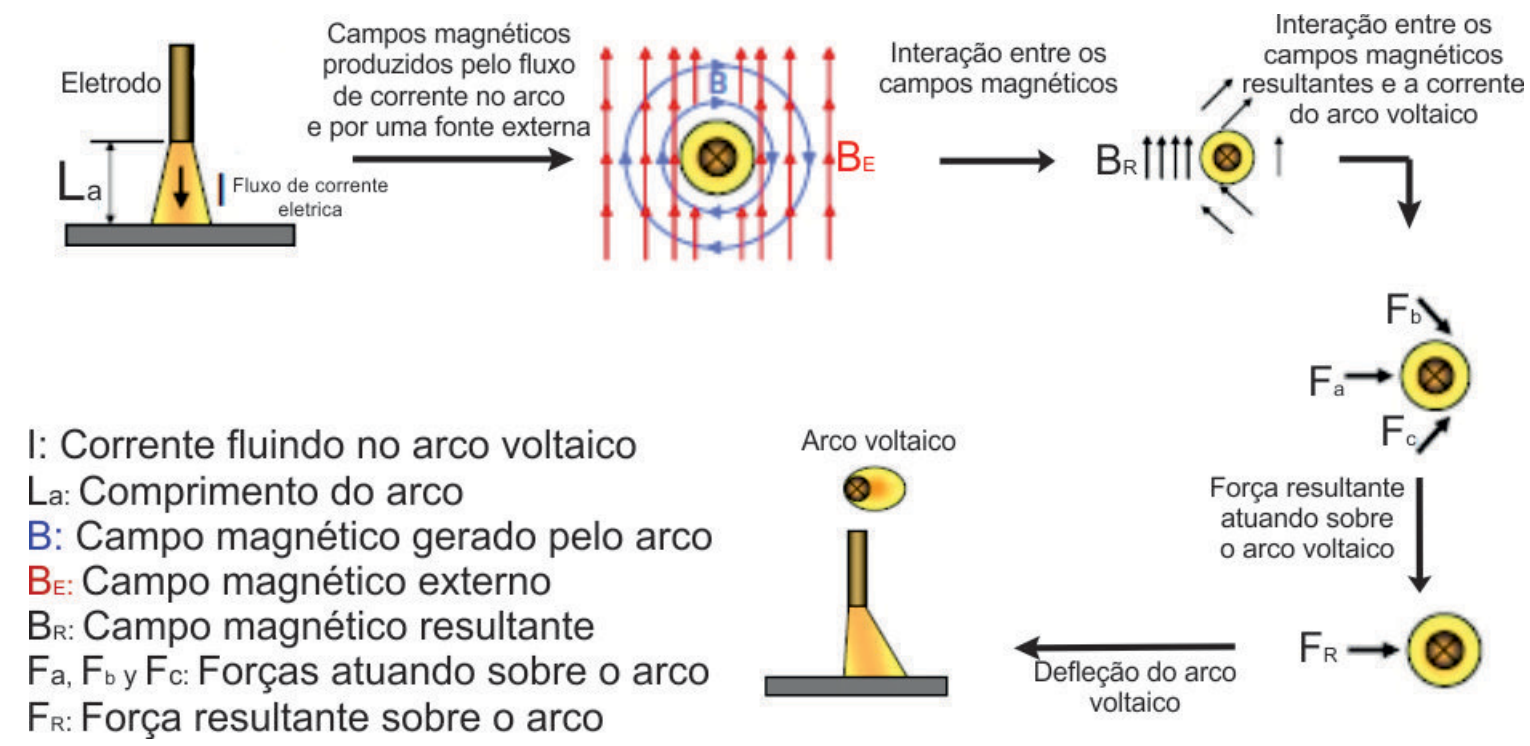

Figura 1. Diagrama explicativo de como atua um campo magnético externo sobre o comportamento do arco voltaico. Adaptado de Reis [15].

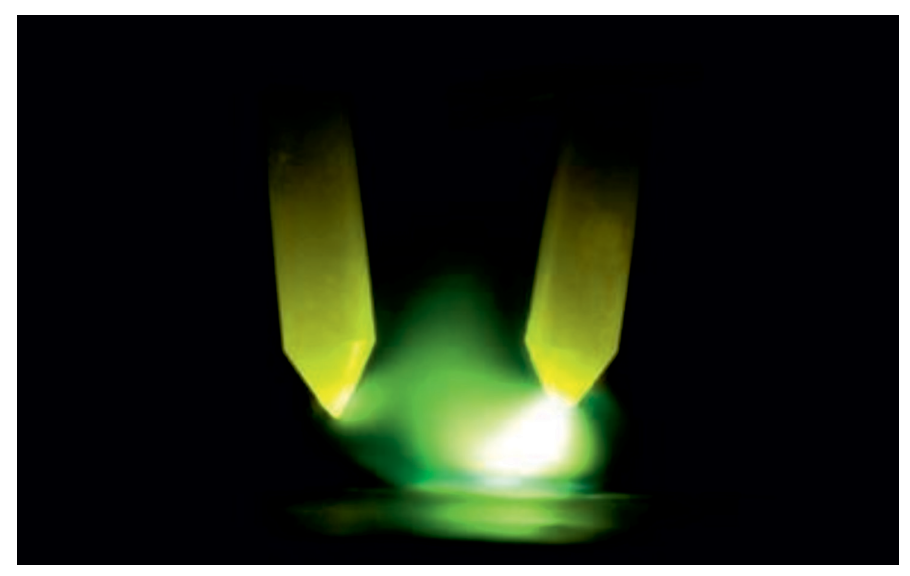

Figura 2. Sopro magnético entre dois arcos voltaicos gerados por eletrodos com a mesma polaridade no processo TIG duplo eletrodo. Adaptado de Schwedersky [16].

A polaridade magnética que o material de adição possui, influencia diretamente a direção que o sopro magnético terá no arco voltaico. Neste sentido, Ueguri et al. [17] menciona que um material de adição que possui a mesma polaridade que o eletrodo provoca um sopro magnético sobre o arco voltaico direcionado para o arame de adição. No caso de ter polaridades diferentes entre o arco e o material de adição, o sopro magnético do arco voltaico ocorrerá no mesmo sentido do avanço da tocha TIG. Para Ueguri et al. [17] essa última configuração é a mais adequada para que dessa forma seja possível conseguir maiores velocidades de soldagem, devido a garantia que o arco formará uma poça de fusão onde o material será depositado.

O processo TIG Hot-Wire destaca-se por conseguir taxas de deposição de pelo menos o dobro do TIG Cold-Wire $[4,6,7,9,10,18]$. Neste sentido, Lucas [6] na Figura 3 mostra que ao aumentar a energia do arco voltaico, a taxa de deposição do processo TIG Hot-Wire aumenta, em especial se é combinado com técnicas de oscilação do arco. Porém, o uso de uma maior energia no arco voltaico implica uma maior diluição [19].

É importante entender que nos revestimentos metálicos, onde o material de adição possui propriedades superiores ao material de base, sempre é preferível que os valores de diluição sejam baixos [18,20]. Com baixos valores de diluição, a composição final do revestimento será próxima à composição do material de adição, obtendo assim propriedades superiores na superfície do material de base. Deve-se ressaltar a necessidade da existência 
Estudo do Processo TIG Hot-Wire com Material de Adição AISI-316L Analisando o Efeito do Sopro Magnético do Arco sobre a Diluição do Cordão de Solda

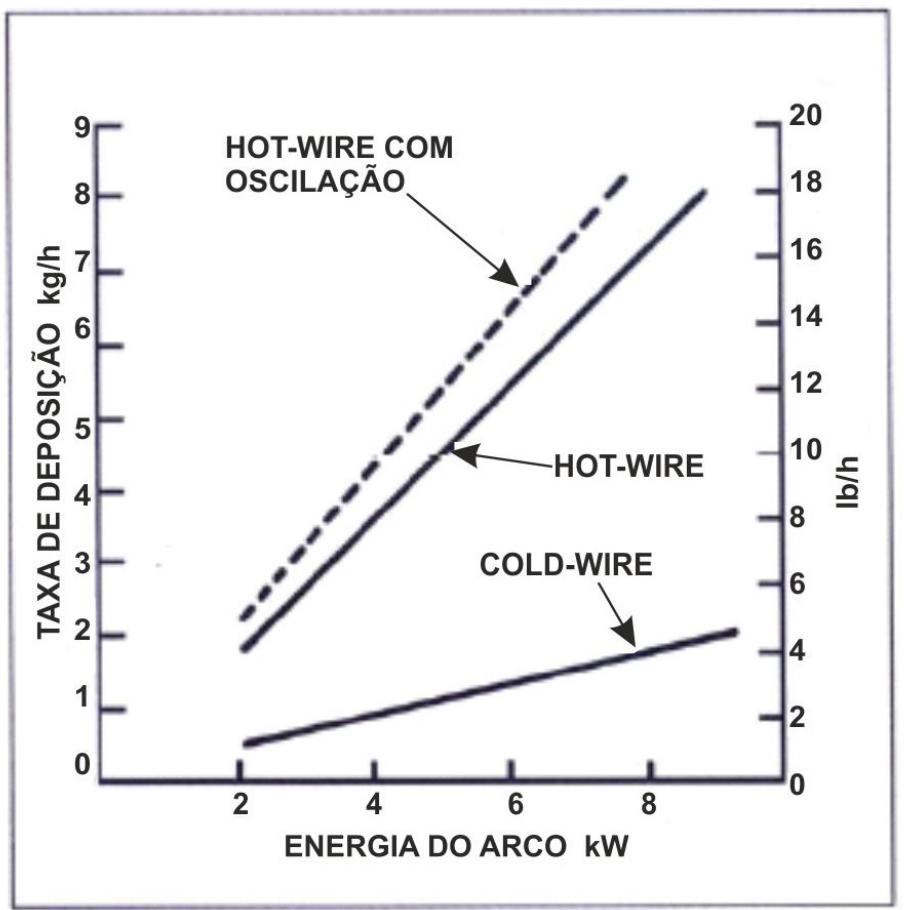

Figura 3. Taxas de deposição que podem ser alcançadas com o processo TIG Hot-Wire comparada com o TIG Cold-Wire. Adaptado de Lucas [6].

de uma quantidade mínima de diluição, para que exista a união metalúrgica entre o revestimento depositado e o metal de base [18]. Portanto, fatores que influenciam a diluição possuem uma grande relevância na produtividade de um processo de soldagem [19].

O objetivo do presente trabalho é fornecer um detalhado estudo do processo TIG Hot-Wire, utilizando o sistema de aquecimento de corrente contínua constante, baseado no princípio de efeito Joule. O objetivo específico é analisar o sopro magnético que a bibliografia descreve e observar o impacto que este possui sobre a produtividade do processo TIG, especialmente sobre a diluição dos cordões de solda.

\section{Materiais e Métodos}

\subsection{Materiais}

As condições foram mantidas constantes em todos os ensaios realizados e são descritas na Figura 4, onde destaca-se a utilização de uma distância eletrodo-peça de $6 \mathrm{~mm}$, ângulo de inclinação do arame de $30^{\circ}$, extensão livre de arame de $26 \mathrm{~mm}, 4 \mathrm{~mm}$ de distância entre o centro do eletrodo e a ponta do arame, além de um ângulo de afiação do eletrodo de $180^{\circ}$ (ponta plana).

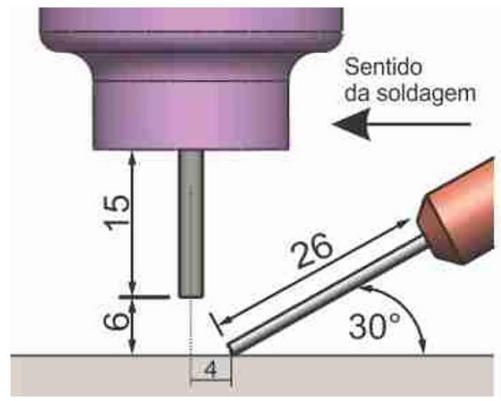

\begin{tabular}{|l|c|}
\hline Eletrodo: & EWTh-2 \\
\hline Diâmetro do eletrodo: & $2,4 \mathrm{~mm}$ \\
\hline Ângulo de afiação: & $180^{\circ}$ \\
\hline Gás de protecão: & Argônio \\
\hline Vazão de gás: & $18 \mathrm{l} / \mathrm{min}$ \\
\hline Material de adição: & $\mathrm{ER} 316 \mathrm{~L}$ \\
\hline Diâmetro de arame: & $1,2 \mathrm{~mm}$ \\
\hline
\end{tabular}

Figura 4. Parâmetros utilizados pelo processo TIG Hot-Wire. 
Foi utilizada uma bancada de ensaios constituída por duas fontes de soldagem da empresa IMC Soldagem, a MINITEC 200 para o aquecimento do arame e a DIGIPLUS A7 a ser usada no processo TIG. A tocha TIG utilizada neste trabalho é do fabricante Abicor Binzel, modelo 27B, refrigerada com água e uma capacidade de trabalho de até $500 \mathrm{~A}$. A tocha foi instalada em um sistema de deslocamento com dois graus de liberdade. Na tocha foi acoplado um direcionador de arame desenvolvido pelo LABSOLDA. O monitoramento da corrente e a tensão tanto do arco voltaico como do aquecimento do arame foi realizado com o sistema de aquisição de dados SAP4. Na Figura 5 mostram-se as configurações dos circuitos elétricos usados para o processo TIG e o sistema de aquecimento do arame para os ensaios realizados segundo a Tabela 1, onde a tocha de soldagem encontra-se conectada ao terminal negativo da fonte, enquanto no sistema de aquecimento do arame foram testados dois circuitos elétricos segundo o apresentado na Figura 5. No caso do circuito $A, 0$ arame está conectado ao terminal negativo da fonte de energia, por tanto teria a mesma polaridade que o processo TIG. Por outro lado, o circuito B está conectado ao terminal positivo da fonte de energia. Todos os ensaios com TIG Hot-Wire foram feitos com os dois circuitos elétricos descritos.

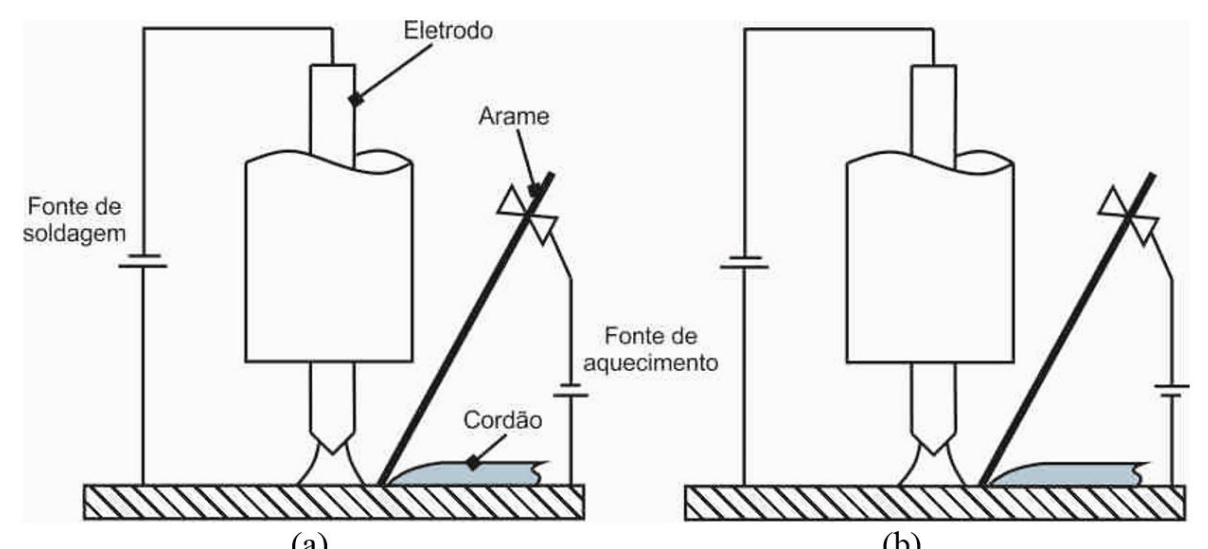

(a)

(b)

Figura 5. Circuitos elétricos usados para os ensaios com o processo TIG Hot-Wire (a) arame conectado no terminal negativo (b) arame conectado no terminal positivo da fonte de energia. Adaptado de Ueguri et al. [17].

Tabela 1. Parâmetros utilizados na comparação entre o processo TIG Hot-Wire e Cold-Wire.

\begin{tabular}{lcc}
\hline \multirow{2}{*}{ Variáveis } & \multicolumn{2}{c}{ Processo TIG } \\
\cline { 2 - 3 } Va [m/min] & Hot-Wire & Cold-Wire \\
& 4,5 & 4,5 \\
& 5,5 & - \\
& 7,5 & - \\
Ic [A] & 8,5 & - \\
I [A] & 180 & - \\
Vs [cm/min] & 260 & 260 \\
\hline
\end{tabular}

Nota: I= corrente do arco voltaico; $\mathrm{Va}=$ velocidade de arame; Vs= velocidade de soldagem; $\mathbf{l}=$ corrente de aquecimento do arame.

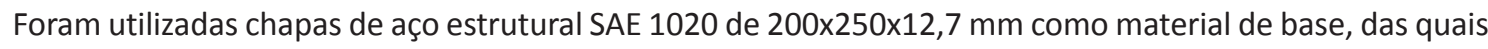
foi removida toda a carepa de óxido ou laminação.

\subsection{Métodos}

Os ensaios foram realizados na posição plana, definindo primeiramente os parâmetros para o processo TIG Hot-Wire usando os dois circuitos descritos na Figura 6, continuando com a comparação com os mesmos parâmetros, mas com o arame frio (Cold-Wire). Dessa forma, a análise vai permitir mostrar as verdadeiras vantagens da utilização do Hot-Wire. 
Estudo do Processo TIG Hot-Wire com Material de Adição AISI-316L Analisando o Efeito do Sopro Magnético do Arco sobre a Diluição do Cordão de Solda

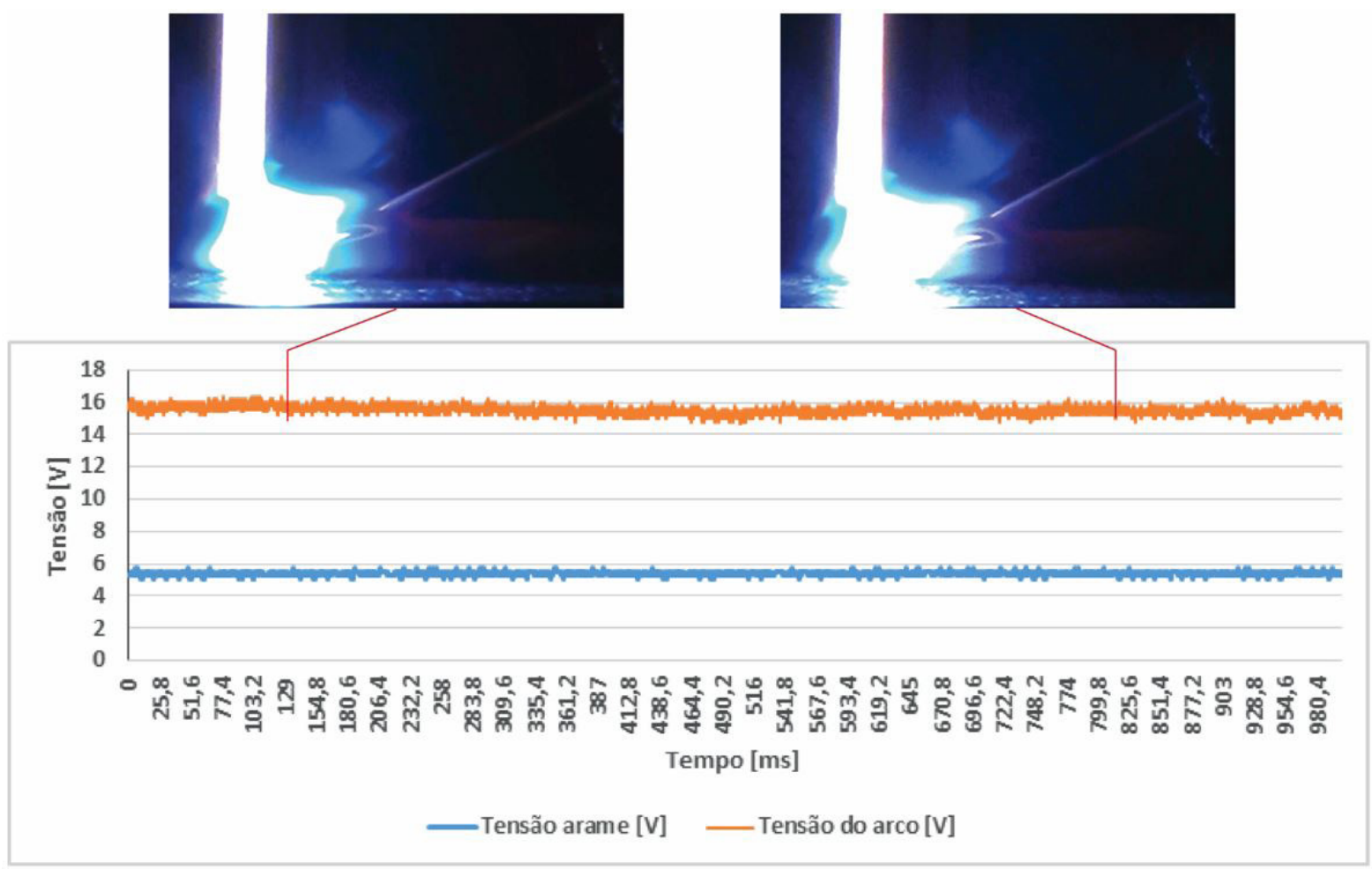

Figura 6. Oscilograma de tensão para o arame de adição e arco voltaico no processo TIG Hot-Wire para uma velocidade de arame de $4,5 \mathrm{~m} / \mathrm{min}$.

A análise do sopro magnético foi realizada por meio de aquisição de imagens para uma mesma corrente de aquecimento, com atenção na estabilidade do processo de soldagem e na aparência superficial do cordão.

A taxa de deposição foi determinada por meio da pesagem dos corpos de prova antes e depois de realizar um cordão de solda, utilizando também os tempos de arco acesso adquiridos para realizar o cálculo.

Destaca-se que todos os ensaios foram repetidos 3 vezes, afim de poder garantir a repetitividade dos resultados obtidos.

\section{Resultados e Discussões}

\subsection{Comparação entre o TIG Hot-Wire e Cold-Wire}

Os ensaios realizados, segundo a Tabela 1, demonstraram que quando ocorre a passagem de uma corrente através do material de adição, o arco voltaico sofre uma evidente deflexão na direção do arame energizado. Isso ocorre como resultado da interação entre os campos magnéticos produzidos entre eles, fato esperado segundo o descrito por Reis [15]. Porém, o sopro magnético observado não afeta a estabilidade do processo de soldagem, ele muda a direção em que atua a pressão do arco e a distribuição da densidade de corrente sobre a região anódica, as quais já não estarão focadas sobre o centro da poça fundida, provocando uma mudança nas correntes convectivas da poça e, portanto, na penetração do cordão [21]. Na Figura 6 observa-se o comportamento das tensões do arco voltaico e do arame de adição durante a realização de um cordão com 4,5 m/min de velocidade de arame, com a configuração A do circuito elétrico (Figura 5). Nesta configuração do circuito elétrico, observa-se que o arco voltaico não possui uma forma simétrica, senão uma forma direcionada para o arame de adição, implicando que a maioria da energia do arco voltaico encontra-se dirigida para o material depositado em vez do metal de base, concordando com o relatado por Ueguri et al. [17].

Os ensaios realizados com Cold-Wire mostraram bastantes instabilidades e respingos sobre o metal de base. Na Figura 7 observa-se uma comparação entre o processo TIG Cold-Wire e Hot-Wire, para uma velocidade de arame de 4,5 m/min, permitindo notar que no caso do Cold-Wire a energia fornecida pelo arco voltaico não é 


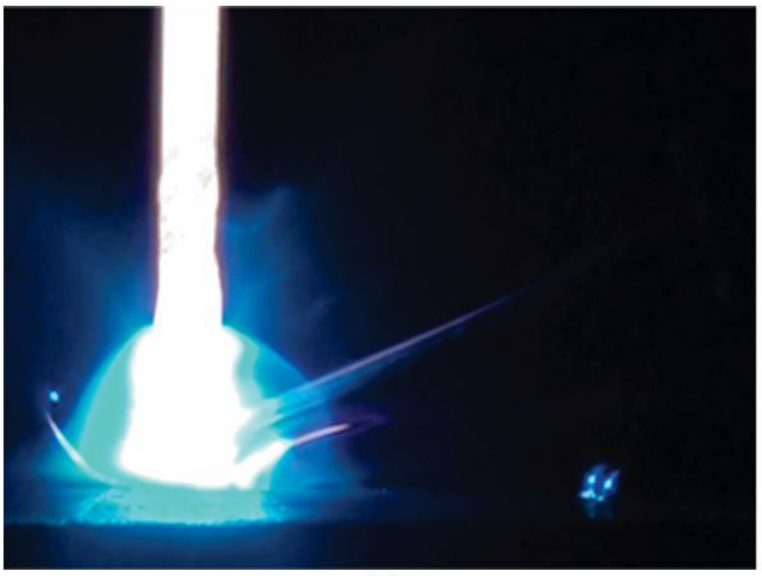

(a)

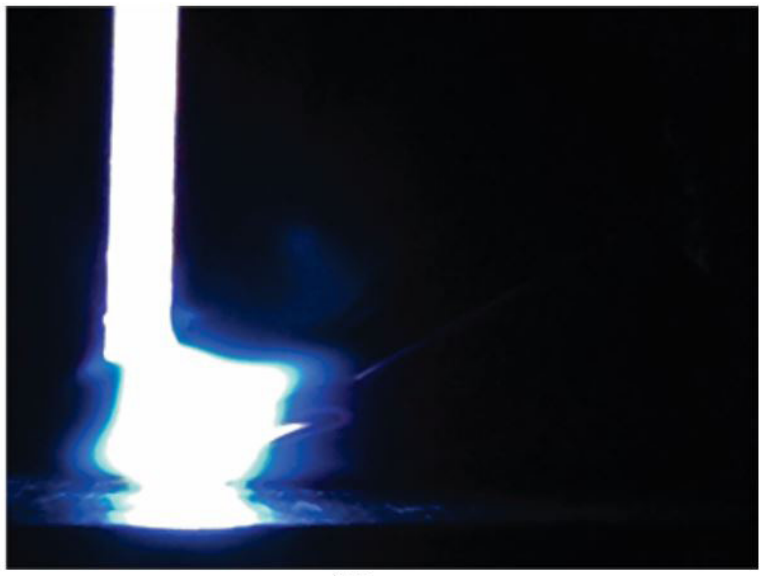

(b)

Figura 7. Arco acesso para o processo TIG (a) Cold-Wire (b) Hot-Wire.

suficiente para conseguir fundir todo o material de adição, implicando que é necessário diminuir a velocidade de arame. Ao não conseguir fundir totalmente o metal de adição, a ponta do arame atravessa o arco voltaico e bate no substrato, provocando uma alteração na distância eletrodo-peça, gerando uma mudança nas propriedades do arco, além de produzir instabilidades e variações na geometria dos cordões de solda.

As melhores qualidades superficiais foram obtidas para as menores velocidades de arame (4,5 e 5,5 m/min), segundo é observado na Figura 8. Desde os 7,5 m/min o aspecto superficial é opaco, enquanto para 8,5 $\mathrm{m} / \mathrm{min}$ a geometria do cordão é irregular, não sendo possível conseguir um cordão repetível para esta última velocidade de arame. No caso do ensaio para 4,5 m/min usando arame frio, na Figura 9 observa-se que o cordão possui uma aparência limpa e geometria irregular, além de notar-se alguns respingos ao redor do mesmo cordão de solda.

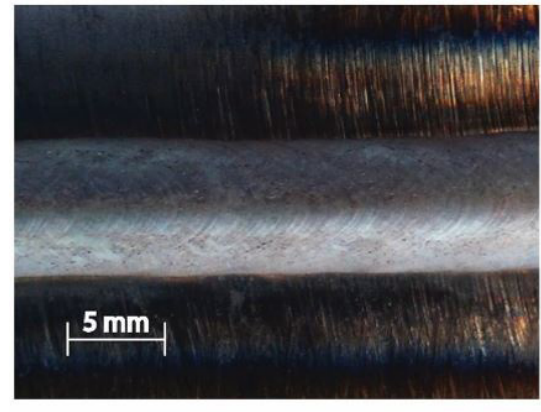

$\mathrm{Va}=4,5 \mathrm{~m} / \mathrm{min}$

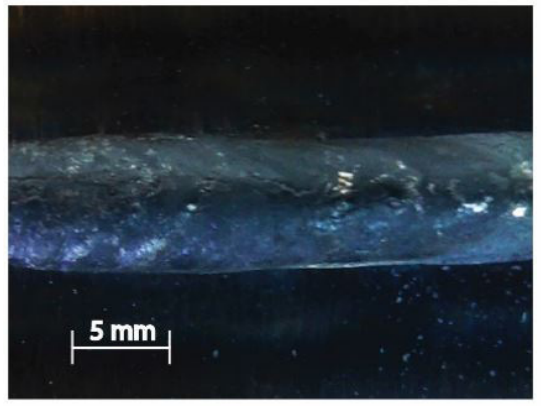

$\mathrm{Va}=7,5 \mathrm{~m} / \mathrm{min}$

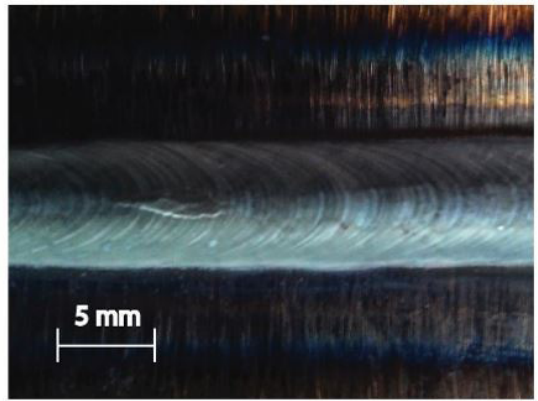

$\mathrm{Va}=5,5 \mathrm{~m} / \mathrm{min}$

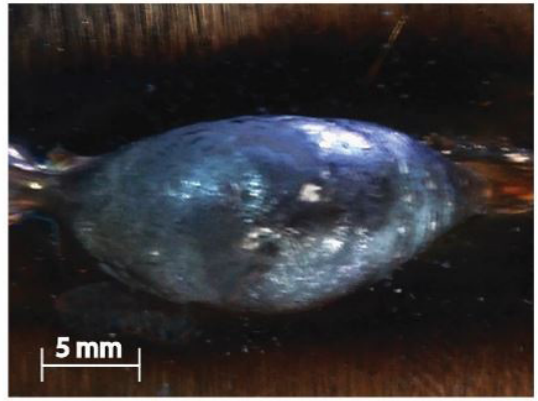

$\mathrm{Va}=8,5 \mathrm{~m} / \mathrm{min}$

Figura 8. Comparação entre as qualidades superficiais para os ensaios com TIG Hot-Wire para quatro níveis de velocidades de arame (Va). 
Estudo do Processo TIG Hot-Wire com Material de Adição AISI-316L Analisando o Efeito do Sopro Magnético do Arco sobre a Diluição do Cordão de Solda

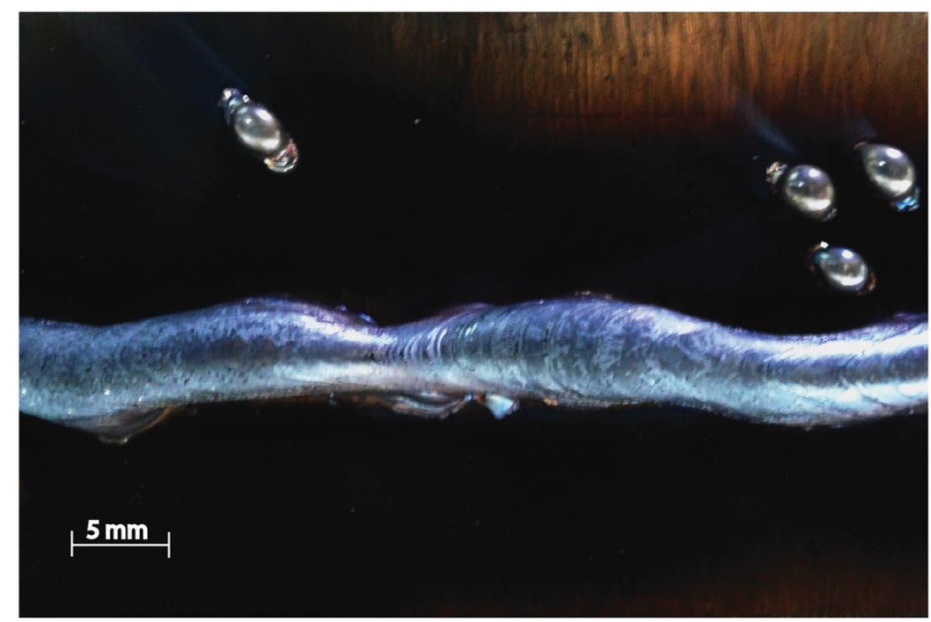

Figura 9. Aparência superficial do cordão de solda para 4,5 m/min de velocidade de arame com o processo TIG Cold-Wire.

Um corte transversal aos cordões realizados permite identificar que a diluição dos ensaios com o processo TIG Hot-Wire encontram-se entre um 12,5 e 1,4\%, dependendo da velocidade de arame utilizada (Figura 10). No caso do ensaio para $8,5 \mathrm{~m} / \mathrm{min}$ não foi realizada uma macrografia, devido ao fato de não ser possível obter cordões repetíveis para analisar. Destacam-se as velocidades de arame conseguidas, para uma corrente de soldagem de $260 \mathrm{~A}$ e uma velocidade de soldagem de $30 \mathrm{~cm} / \mathrm{min}$. Por outro lado, os cordões obtidos para as velocidades de arame de $5,5 \mathrm{~m} / \mathrm{min}$ e $7,5 \mathrm{~m} / \mathrm{min}$ possuem um excessivo ângulo de molhamento, embora não sejam descartados para uma aplicação de revestimento usando oscilação da tocha de soldagem, para reduzir o reforço e a diluição [22]. Os melhores resultados tanto em aparência superficial (Figura 9) como em geometria do cordão foram para $4,5 \mathrm{~m} / \mathrm{min}$ de velocidade de arame.

Os ensaios com o processo TIG Cold-Wire com 4,5 m/min de velocidade de arame apresentaram uma diluição similar à do Hot-Wire para 7,5 m/min (Figura 11), porém, a instabilidade do processo e a falta de fusão do material de adição fazem inviáveis a utilização dessa velocidade de arame com o Cold-Wire. Nos trabalhos de Silva et al. [2] e Miranda [3] foram conseguidos cordões com uma diluição de aproximadamente $16 \%$, usando o processo TIG

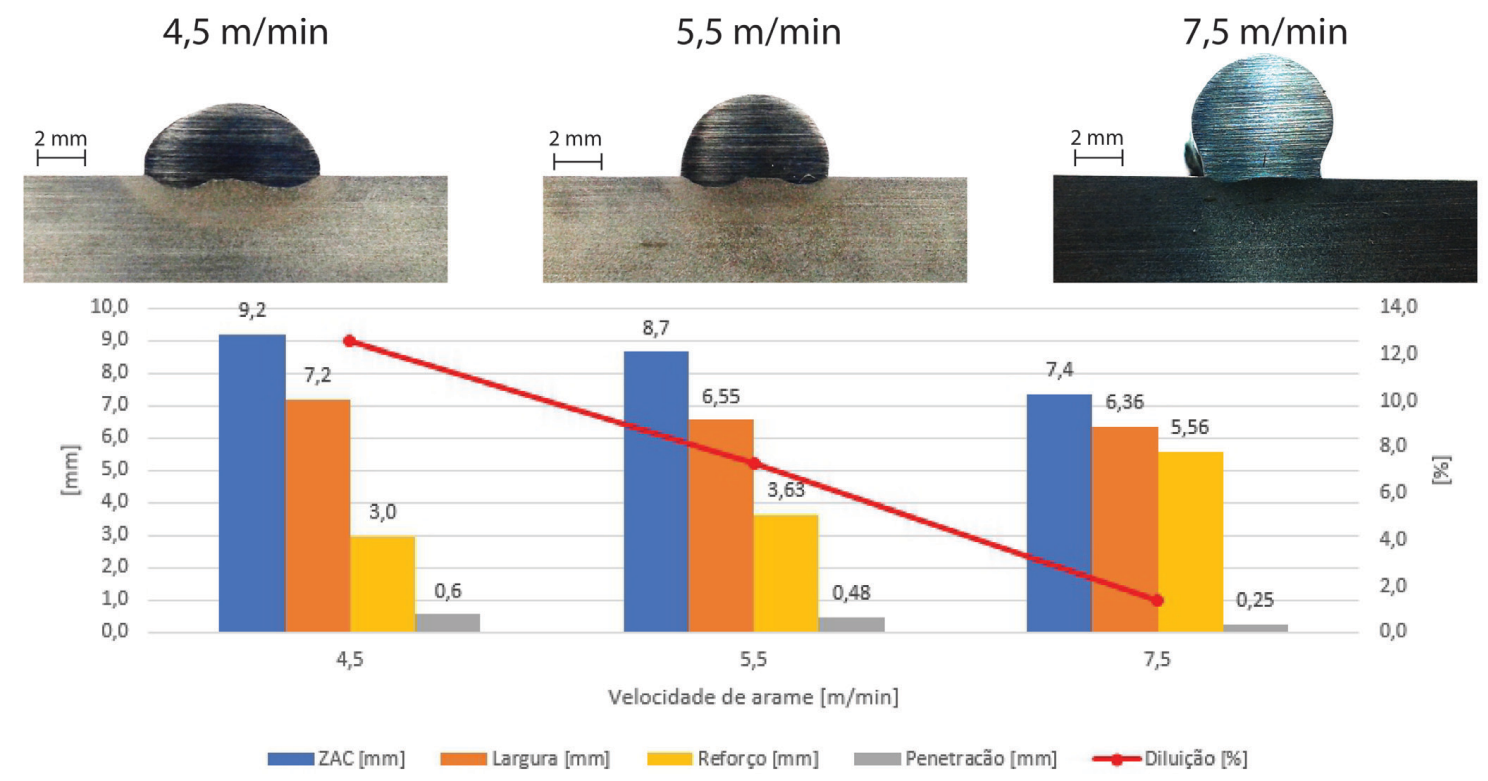

Figura 10. Análise geométrica dos cordões realizados com o processo TIG Hot-Wire. 


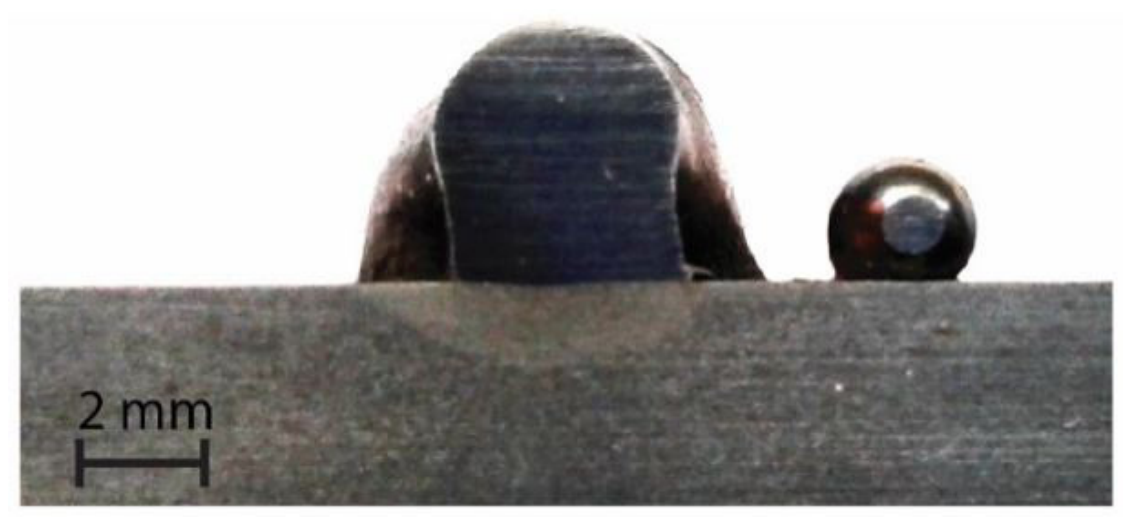

Figura 11. Macrografía do ensaio para 4,5 m/min de velocidade de arame com o processo TIG Cold-Wire.

Cold-Wire com $6 \mathrm{~mm}$ de comprimento do arco e $6 \mathrm{~m} / \mathrm{min}$ de velocidade de alimentação. Diluições menores foram obtidas, chegando até um $10 \%$ para $14 \mathrm{~mm}$ de comprimento de arco, porém, uma alta convexidade e falta de fusão dos cordões de solda foram evidenciadas [2]. Neste sentido o TIG Hot-Wire demonstra ser superior ao Cold-Wire, conseguindo menores diluições e maiores velocidades de alimentação de arame. Uma maior produtividade não foi obtida com o processo TIG Cold-Wire, além de não trabalhar com velocidades de arame acima de 4,5 m/min, portanto a análise desse processo não foi continuada.

Na Figura 12 encontram-se as taxas de deposição para as diferentes velocidades de arame estudadas com o processo Hot-Wire, onde evidentemente, para uma velocidade de arame de 7,5 m/min se tem a maior taxa de deposição $(4 \mathrm{~kg} / \mathrm{h}$ ) para a mesma potência de aquecimento do arame de $1 \mathrm{~kW}$ aproximadamente. Comparando os dados obtidos com o gráfico de Lucas [6] da Figura 3, aprecia-se, que para uma potência de 3,5 kW do arco voltaico, a taxa de deposição esperada é de $3 \mathrm{~kg} / \mathrm{h}$ aproximadamente, resultado obtido pelo presente estudo para velocidades de arame de 4,5 e 5,5 m/min.

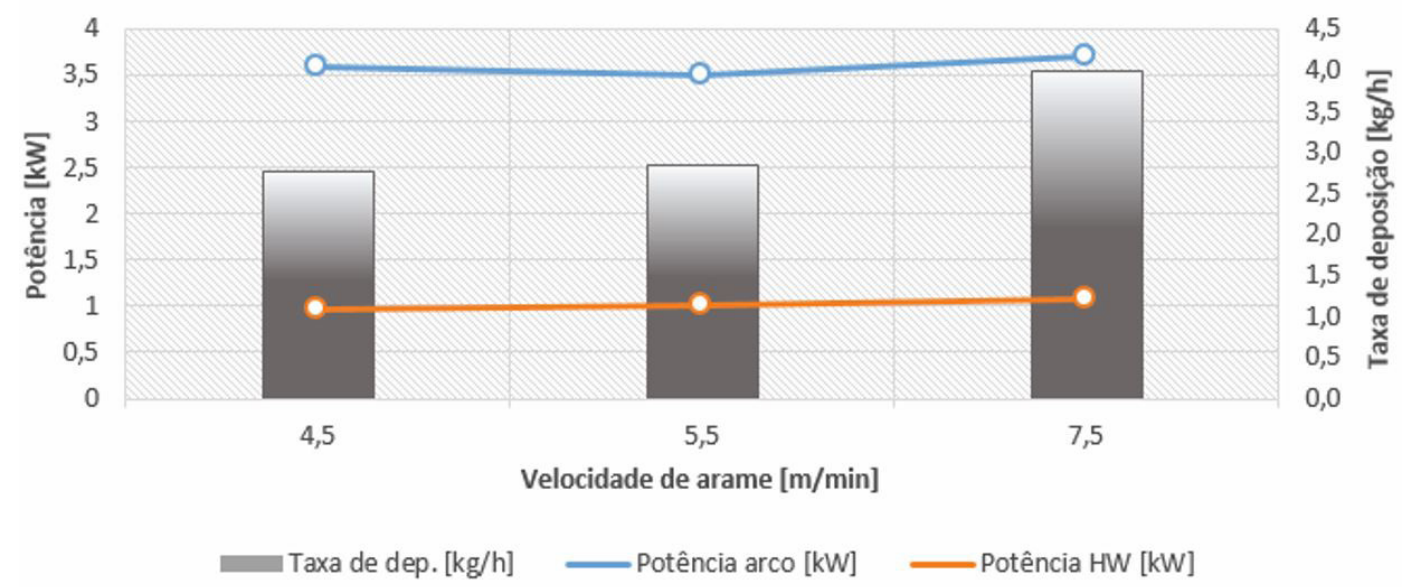

Figura 12. Analise da taxa de deposição em comparação com a potência utilizada para o processo TIG Hot-Wire.

Dessa primeira análise observou-se que efetivamente o processo TIG Hot-Wire é mais produtivo que o processo TIG com arame frio, além de possuir níveis de diluição menores. De fato, os resultados obtidos concordam com a bibliografia referenciada. O sopro magnético produzido pelo arame quente sobre o arco voltaico, permite que as propriedades do arco (pressão e densidade de corrente) não sejam concentradas no centro da poça de fusão, favorecendo a diminuição da penetração e o aquecimento do material de adição. Além, a maior quantidade de material depositado é produto tanto do aquecimento do arame pela fonte externa de corrente continua constante, quanto do próprio arco voltaico direcionado para o arame pelo sopro magnético. Portanto, quanto maior for a quantidade de material depositado menor será a diluição do cordão, para uma mesma corrente do arco voltaico. 
Estudo do Processo TIG Hot-Wire com Material de Adição AISI-316L Analisando o Efeito do Sopro Magnético do Arco sobre a Diluição do Cordão de Solda

\subsection{Efeito da polaridade do arame de adição sobre o sopro magnético no arco voltaico e no comportamento do cordão de solda}

No item anterior foi utilizado o circuito elétrico A (Figura 5), configuração que segundo Ueguri et al. [17] faz com que a energia do arco voltaico seja direcionada por completo para o material de adição, como se observou na Figura 7. Por outro lado, se fosse a configuração B do circuito elétrico da Figura 5 , o arco voltaico se direcionaria no mesmo sentido do avanço da tocha TIG. Segundo Ueguri et al. [17] essa condição é desejável para se trabalhar com altas velocidades de soldagem, porque assim é garantida a formação de uma poça fundida por onde o material será depositado. Porém, segundo os ensaios realizados, foi demonstrado que não seria factível usar essa polaridade permanentemente para aquecer o arame. Na Figura 13 observa-se como o arco voltaico afasta-se do material de adição, devido ao campo magnético gerado pelo fluxo de corrente que circula pelo material de adição, isto implica que não existe contato entre o arco voltaico e o arame de adição, fazendo com que a transferência de energia do arco para o material de aporte seja mínima, provando que não ocorre aderência entre o material depositado e o metal de base, ou seja, diluição zero.

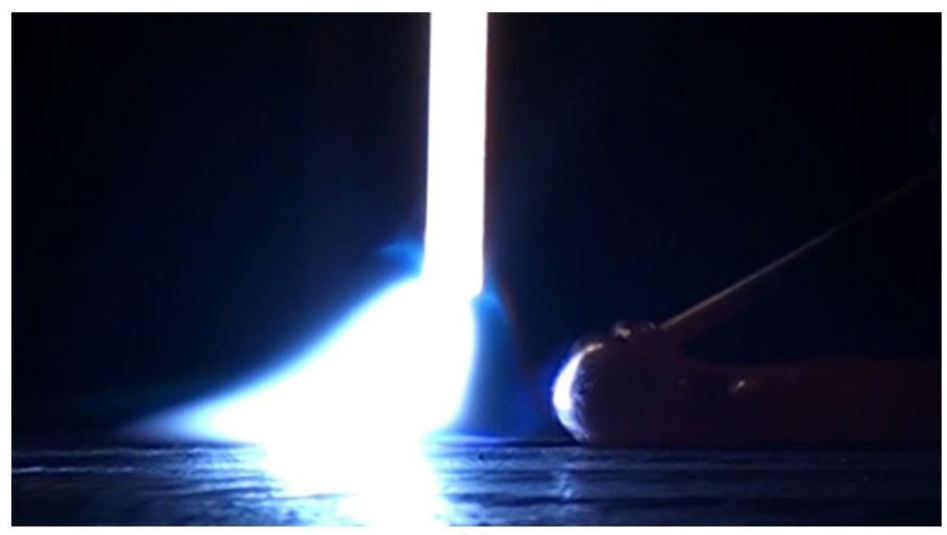

(a)

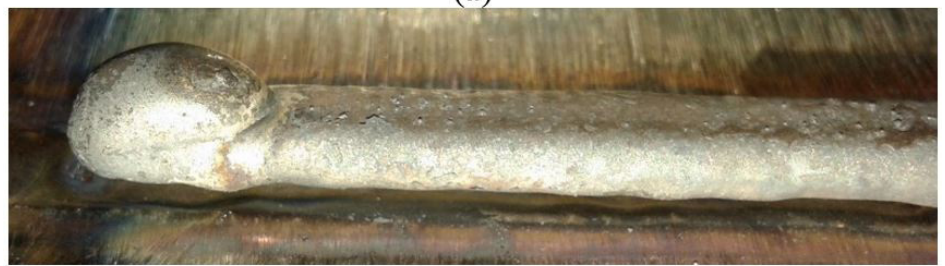

(b)

Figura 13. Processo TIG Hot-Wire com arame de adição conectado ao terminal positivo da fonte de energia; (a) Hot-Wire com 4,5 m/min de velocidade de alimentação; (b) Aparência superficial do cordão sem nenhuma diluição com o metal de base.

Na tentativa de melhorar a transferência de energia entre o arco voltaico e o material de adição, aumentou-se a velocidade de arame para 7,5 m/min, alcançando-se o objetivo proposto, porém, a energia proporcionada pelo arco não é suficiente para conseguir um cordão de solda satisfatório. Na Figura 14 observa-se o mencionado, onde o arco voltaico não consegue fundir todo o material de adição, fazendo com que o arame se desvie do centro do cordão de solda, originando um aspecto superficial inadequado.

O resultado apresentado na Figura 14 dista consideravelmente dos cordões realizados com os mesmos parâmetros, mas com o arame negativo (circuito A). O sopro magnético provocado pelo circuito B não favoreceu um aumento na taxa de deposição, porém foi evidenciada a formação de uma poça fundida na frente do material depositado, o qual concorda com o descrito por Ueguri et al. [17]. Neste sentido, as tecnologias de aquecimento do arame que usam as duas polaridades da corrente, descritas nos trabalhos de Burt [14] e o CCWJ [8], seriam uma melhor alternativa que usar o circuito B permanentemente, dessa forma as vantagens de cada polaridade seriam melhor aproveitadas. 


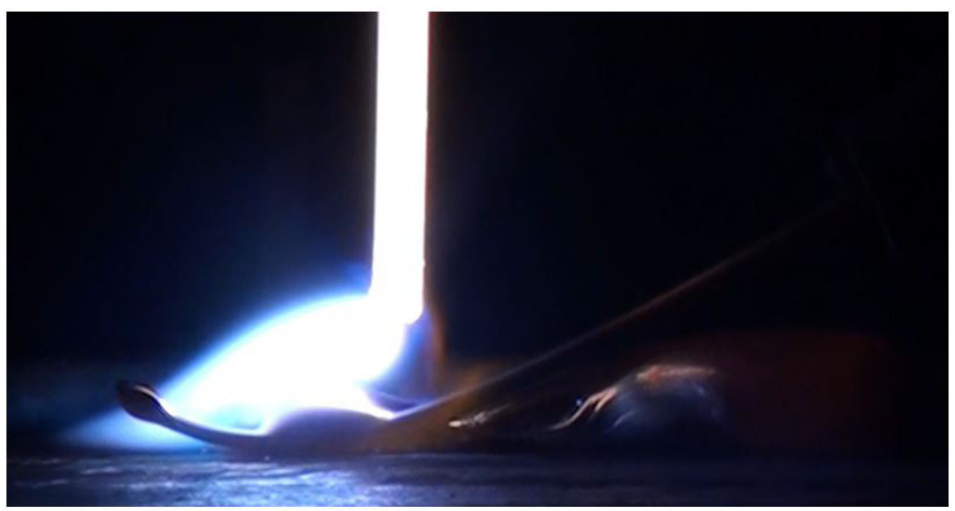

(a)

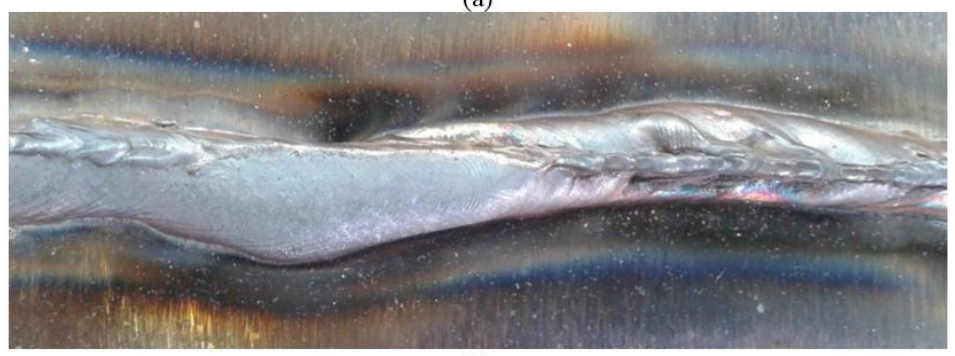

(b)

Figura 14. Resultado dos ensaios com TIG Hot-Wire com arame de adição conectado ao terminal positivo da fonte de energia; (a) Arco voltaico acesso; (b) Aparência superficial do cordão realizado.

\section{Conclusões}

Por meio dos resultados e das análises realizadas entre os processos TIG Cold-Wire e Hot-Wire com as mudanças na polaridade da corrente para o aquecimento do material de adição, é possível concluir que:

- Efetivamente o processo TIG com adição de arame quente (Hot-Wire) possui uma maior taxa de deposição, em comparação ao uso de arame frio (Cold-Wire). As taxas de deposição obtidas são comparáveis com as conseguidas por outros autores da bibliografia referenciada.

- No caso dos ensaios realizados com o processo TIG Cold-Wire não foi possível obter um cordão de solda aceitável, para as velocidades usadas com Hot-Wire. Portanto fica evidente a maior produtividade do Hot-Wire frente ao Cold-Wire.

- A menor porcentagem de diluição conseguida por meio do processo TIG Hot-Wire foi de um $2 \%$ aproximadamente, para uma velocidade de arame de $7,5 \mathrm{~m} / \mathrm{min}$. Enquanto para uma velocidade de $5,5 \mathrm{~m} / \mathrm{min}$ a diluição obtida foi de um 7\%. Porém, os ângulos de molhamento dos cordões para essas velocidades de arame foram muito altos, dificultando uma aplicação direta para operações de revestimento. Segundo a bibliografia referenciada, a utilização de oscilação da tocha de soldagem resolveria o problema.

- Durante a realização dos ensaios com o processo TIG Hot-Wire foi possível observar que o arco voltaico sofre um sopro magnético, o qual depende da polaridade da conexão do arame de adição à fonte de energia. O sopro magnético observado tem uma influência na direção e distribuição das propriedades do arco sobre a poça fundida e do material de adição. Neste sentido o sopro magnético favorece uma maior transferência de energia para o arame quente desde o arco voltaico, além de diminuir a pressão do arco no centro da poça fundida, implicando um aumento na taxa de deposição e uma diminuição na diluição do cordão de solda.

- Quando o arame de adição é conectado ao terminal positivo da fonte de energia, o arco voltaico sofre um sopro magnético na mesma direção do avanço da tocha de soldagem, havendo pouca transferência de energia entre $\mathrm{o}$ arco voltaico e o arame de adição, fazendo com que o material não seja totalmente fundido. Porém, foi evidenciado que esta polaridade garante a formação da poça fundida. 
Estudo do Processo TIG Hot-Wire com Material de Adição AISI-316L Analisando o Efeito do Sopro Magnético do Arco sobre a Diluição do Cordão de Solda

\section{Agradecimentos}

Os autores apresentam seus agradecimentos, pela colaboração financeira a FIC-R 2012 e à Vicerrectoría de Investigación, Innovación y Postgrado da Universidade de Antofagasta através do projeto Fomento à Pesquisa.

\section{Referências}

[1] Vergara VM. Inovação do equipamento e avaliação do processo Plasma de Arco Transferido Alimentado com Pó (PTAP) para soldagem fora de posição [tese de doutorado]. Florianópolis: Universidade Federal de Santa Catarina; 2005.

[2] Silva CC, Miranda EC, Motta MF, Miranda HC, Farias JP. Influence of arc length on dilution and weld bead geometry of Ni-Based alloy using GTAW process with cold wire feed. In: Associação Brasileira de Ciências Mecânicas. Proceedings of the 20th International Congress of Mechanical Engineering; 2009 November 15-20; Gramado, Brazil. Rio de Janeiro: ABCM; 2009. p. 1-10.

[3] Miranda ED. Estudo exploratório de parâmetros de soldagem de revestimentos com ligas de níquel empregando o processo TIG com alimentção de arame frio [dissertação de mestrado]. Fortaleza: Universidade Federal do Ceará; 2009.

[4] Henon BK. Advances in automatic hot wire GTAW (TIG) welding [página da internet]. Pacoima: Arc Machines Inc.; 2015 [acesso em 14 set. 2015]. Disponivel em: http://www.arcmachines. com/news/case-studies/advances-automatic-hot-wire-gtawtig-welding

[5] Shinozaki K, Yamamoto M, Mitsuhata K, Nagashima T, Kanazawa $\mathrm{T}$, Arashin $\mathrm{H}$. Bead formation and wire temperature distribution during ULTRA-HIGH-SPEED GTA welding using pulse-heated hot-wire. Welding in the World. 2011;55(3-4):12-18. http:// dx.doi.org/10.1007/BF03321281.

[6] Lucas W. TIG and plasma welding: process techniques, recommended practices and applications. Cambridge: Abington Publishing; 1990.

[7] Shinozaki K, Yamamoto M, Nagamitsu Y, Uchida T, Mitsuhata K, Nagashima T, et al. Melting phenomenon during ultra-high-speed GTA welding method using pulse-heated hot-wire. Quarterly Journal of the Japan Welding Society. 2009;27(2):22s-26s. http:// dx.doi.org/10.2207/qjjws.27.22s.

[8] Canadian Center for Welding and Joining - CCWJ. HWGTAW steel 100AR DCEN CC GTAW with hot-wire addition. [página da internet]. Alberta: CCWJ; 2012 [acesso em 03 fev. 2016]. Disponivel em: https://www.ualberta.ca/ ccwj/videos/pages/ Intro\%20High\%20Speed/06_Alternative\%20Welding\%20 Processes/HWGTAW_Steel_100Ar_DCEN\%20CC\%20GTAW\%20 with\%20Hot-Wire\%20Addition_001/

[9] Lv SX, Tian XB, Wang HT, Yang SQ. Arc heating hot wire assisted arc welding technique for low resistance welding wire. Science and Technology of Welding and Joining. 2007;12(5),431-435. http://dx.doi.org/10.1179/174329307X213828.
[10] Lv SX, Xu ZW, Wang HT, Yang SQ. Investigation on TIG cladding of copper alloy on steel plate. Science and Technology of Welding and Joining. 2008;13(1):10-16.

[11] Jenney CL, O'Brien A, editores. Welding Handbook: welding science and technology. 8th ed. Miami: American Welding Society; 1991. (vol. 1).

[12] Reis RP, Souza D, Scotti A. Models to describe plasma jet, arc trajectory and arc blow formation in arc welding. Welding in the World. 2011;55(3-4),24-32.

[13] Lancaster JF. The physics of welding. Oxford: Pergamon Press; 1986.

[14] Burt A. Sustainability through innovative welding processes in automated applications. Mündersbach: EWM Hightec Welding $\mathrm{GmbH}$; 2013. p. 4.

[15] Reis RP. Assessment of low current tandem GMAW processes with waveform control and with aid of laser beam. Uberlândia: Universidade Federal de Uberlândia; 2009.

[16] Schwedersky MB. Estudo e desenvolvimento do processo TIG duplo electro. Florianópolis: Universidade Federal de Santa Catarina; 2015.

[17] Ueguri S, Tabata Y, Shimizu T, Mizuno T. Control of deposition rates in hot wire TIG welding. Welding International. 1987;1(4):736742. http://dx.doi.org/10.1080/09507118709451085.

[18] Silva IG. Estudo da influência dos parâmetros de soldagem de revestimento resistente à corrosão pelo processo TIG modalidade arame quente na diluição [dissertação de mestrado]. Curitiba: Universidade Tecnológica Federal do Paraná; 2007.

[19] Fratari RQ, Schvartzman MMAM, Scotti A. Otimização dos parâmetros de tecimento para confeção de amanteigamento em chapas de aço ao carbono pelo processo TIG com arame AWS ER309L. Soldagem e Inspeção, São Paulo. 2010;15(3):209217.

[20] Dupont JN, Marder AR. Dilution in single pass arc welds. Metallurgical and Materials Transactions. B, Process Metallurgy and Materials Processing Science. 1996;27B(3):481-489. http:// dx.doi.org/10.1007/BF02914913.

[21] Kou S. Welding metallurgy. New Jersey: John Wiley; 2003.

[22] Miranda ED, Silva CC, Motta MF, Miranda HC, Farias JP. Avaliação do uso do tecimento sobre o nível de diluição e geometria do cordão de solda na soldagem TIG com alimentação automatica de arame frio. Soldagem \& Inspeção. 2015;20(2):180-190. http:// dx.doi.org/10.1590/0104-9224/SI2002.06. 